OPEN ACCESS

Edited by:

Dora ll'yasova,

Duke University, United States

Reviewed by:

Bruna Lancia Zampieri,

Hospital Israelita Albert Einstein, Brazil

Xuanye Cao,

University of Texas MD Anderson

Cancer Center, United States

${ }^{*}$ Correspondence:

Jiabi Qin

qinjiabi123@163.com

Ping Zhu

zhuping6588@163.com

Specialty section

This article was submitted to

Children and Health,

a section of the journal

Frontiers in Pediatrics

Received: 29 September 2021 Accepted: 08 December 2021

Published: 02 February 2022

Citation:

Song X, Liu Y, Wang T, Zhang S, Sun M, Shu J, Wei J, Diao J, Li J, Li Y,

Chen L, Zhu P and Qin J (2022) Association of Maternal Dietary Habits and MTHFD1 Gene Polymorphisms With Ventricular Septal Defects in Offspring: A Case-Control Study.

Front. Pediatr. 9:785440

doi: $10.3389 /$ fped.2021.785440

\section{Association of Maternal Dietary Habits and MTHFD1 Gene Polymorphisms With Ventricular Septal Defects in Offspring: A Case-Control Study}

\author{
Xinli Song ${ }^{1}$, Yiping Liu ${ }^{1}$, Tingting Wang ${ }^{2}$, Senmao Zhang ${ }^{1}$, Mengting Sun ${ }^{1}$, Jing Shu ${ }^{1}$, \\ Jianhui Wei ${ }^{1}$, Jingyi Diao ${ }^{1}$, Jinqi $\mathrm{Li}^{1}$, Yihuan $\mathrm{Li}^{1}$, Letao Chen ${ }^{1}$, Ping Zhu ${ }^{3 *}$ and \\ Jiabi Qin ${ }^{1,2,3,4 *}$ \\ ${ }^{1}$ Department of Epidemiology and Health Statistics, Xiangya School of Public Health, Central South University, Changsha, \\ China, ${ }^{2}$ National Health Committee Key Laboratory of Birth Defect for Research and Prevention, Hunan Provincial Maternal \\ and Child Health Care Hospital, Changsha, China, ${ }^{3}$ Guangdong Academy of Medical Sciences, Guangdong Cardiovascular \\ Institute, Guangdong Provincial People's Hospital, Guangzhou, China, ${ }^{4}$ Hunan Provincial Key Laboratory of Clinical \\ Epidemiology, Changsha, China
}

Objectives: This study aimed at assessing the association between maternal methylenetetrahydrofolate dehydrogenase 1 (MTHFD1) gene polymorphisms, maternal dietary habits, and their interactions with the risk of ventricular septal defects (VSD) in offspring.

Methods: From November 2017 to March 2019, a case-control study comprising 360 mothers of VSD cases and 504 mothers of healthy infants was conducted in Han Chinese populations. The main exposures of interest were maternal dietary habits in early pregnancy and MTHFD1 gene polymorphisms. Logistic regression models were used to estimate the main effects and interaction effects.

Results: It was observed that maternal excessive intake of pickled vegetables (aOR $=$ 1.85, 95\%Cl: 1.45-2.37), smoked foods (aOR $=1.93,95 \% \mathrm{Cl}: 1.48-2.51)$, barbecued foods ( $\mathrm{aOR}=1.74,95 \% \mathrm{Cl}: 1.28-2.36)$, and fried foods (aOR $=1.68,95 \% \mathrm{Cl}: 1.30-2.17)$ were associated with a higher risk of VSD in offspring, whereas maternal excessive intake of fresh meat $(\mathrm{aOR}=0.61,95 \% \mathrm{Cl}$ : 0.47-0.79), fish and shrimp $(\mathrm{aOR}=0.29,95 \% \mathrm{Cl}$ : 0.23-0.38), fresh eggs $(\mathrm{aOR}=0.54,95 \% \mathrm{Cl}$ : 0.42-0.70), fresh fruits or vegetables (aOR $=0.44,95 \% \mathrm{Cl}: 0.33-0.60)$, soy foods (aOR $=0.65,95 \% \mathrm{Cl}: 0.53-0.80)$, and milk products $(\mathrm{aOR}=0.49,95 \% \mathrm{Cl}: 0.40-0.59)$ could contribute significantly to a lower risk of VSD in offspring. Furthermore, the genetic polymorphisms of maternal MTHFD1 gene at rs1950902 (GA vs. GG: aOR $=0.67,95 \% \mathrm{Cl}: 0.50-0.90$ ) and rs2236222 (GG vs. AA: aOR $=2.75,95 \% \mathrm{Cl}: 1.57-4.83$ ) were significantly associated with the risk of VSD in offspring. In addition, there was a significant interaction effect between maternal dietary habits and MTHFD1 gene polymorphisms on the risk of VSD. 
Conclusions: Maternal dietary factors, MTHFD1 genetic polymorphisms, and their interactions were all associated with the risk of VSD in offspring. However, further research in diverse ethnic populations and with a larger sample size is warranted to corroborate our findings.

Trial Registration: Registered in Chinese Clinical Trial Registry Center; registration number, ChiCTR1800016635; registration date, 06/14/2018 (Retrospectively registered); URL of trial registry record, https://www.chictr.org.cn/showproj.aspx?proj=28300.

Keywords: ventricular septal defects, MTHFD1 gene, interaction effects, case-control study, dietary habits

\section{INTRODUCTION}

Congenital heart disease (CHD) refers to a group of anatomic heart and great vessel malformations that arise during the embryologic development of the fetus. CHD is one of the most prevalent birth defects, affecting around 2.50 out of every 1,000 births in China (1), and it imposes a substantial burden on society and families, making it a serious public health issue. Ventricular septal defects (VSD) are the most common congenital cardiac malformations (2). It is generally understood that folic acid supplementation is the most effective large-scale strategy to date for decreasing CHD, particularly for conotruncal defects (CTD) and VSD $(3,4)$. Also, it has been proven that CTD and VSD likely share some risk factors (5); both are likely associated with folate metabolism (6). However, the underlying etiology of VSD, which comprises a simple or complicated combination of genetic, environmental, and lifestyle factors, is not fully known.

Early work in our laboratory and other literature reveal that genetic polymorphisms of genes related to the maternal folate-homocysteine metabolism pathway, such as methylenetetrahydrofolate reductase (MTHFR) (7), cystathionine beta synthase (CBS) (8), and methionine synthase (MS) (9), are closely associated with the development of CHD. In this study, the gene of interest is methylenetetrahydrofolate dehydrogenase 1 (MTHFD1), which has gotten less attention than the abovementioned genes despite its importance in one-carbon folate metabolism. The MTHFD1 gene encodes a trifunctional enzyme that includes 5,10-methylenetetrahydrofolate dehydrogenase, 5,10-methenyltetrahydrofolate cyclohydrolase, and 10-formyltetrahydrofolate synthetase (10). MTHFD1 catalyzes three sequential reactions that interconvert tetrahydrofolate (THF) to 5,10-methylenetetrahydrofolate (5,10-methylene THF) $(10,11)$. Of note, 5,10-methylene THF has a wide range of applications, including purine, thymidylate, and serine synthesis as well as regenerating methionine from homocysteine in the methylation cycle $(12,13)$. Because of the significance of the MTHFD1 enzyme, it is assumed that abnormal expression or decreased activity could cause a folatehomocysteine imbalance and interfere with DNA synthesis. Experiments indicate that the MTHFD1 gene with mutant genotypes expresses less stable in vitro and less active in vivo MTHFD1 protein, disrupting de novo purine and DNA synthesis $(14,15)$. Furthermore, previous epidemiologic studies show that functional non-synonymous single nucleotide polymorphism
(SNPs) of the MTHFD1 gene, such as MTHFD1 rs2236225 (G1958A; R653Q) and MTHFD1 rs1950902 (C401T; R134K), are associated with an increased risk of $\mathrm{CHD}$, albeit the associations are controversial $(14,16,17)$. To provide more epidemiological data, this study focuses on VSD, including conotruncal VSD (CTD cases with VSD), and investigates the association between maternal SNPs of the MTHFD1 gene and the risk of VSD in Chinese populations.

Dietary habits, as a crucial modifiable influencing factor, are shown to influence pregnancy outcomes, including birth defects. To date, the majority of attention has been focused on the role of nutrients or food items alone or in conjunction with dietary patterns in the development of CHD (18-20). However, given the diversity in socioeconomic status, races, geographic location, and culture, it is apparent that dietary factors vary by population. As a result, this study focuses on the associations between maternal dietary habits and the risk of VSD in offspring, which is helpful for accurate CHD prevention. In addition, previous study has discovered a substantial relationship between dietary habits and serum and red blood cell folate levels $(21,22)$. According to Christensen's study, low dietary folate interacts with MTHFD1 synthetase deficiency in mice, a model for the R653Q mutation, to significantly increase the risk of birth defects (22). Thus, we hypothesize that the maternal MTHFD1 gene and dietary habits combine to increase the risk of VSD. Based on the abovementioned background, we performed a hospital-based, case-control study in Han Chinese populations to (1) assess the association between maternal dietary habits in the first-trimester pregnancy and the risk of VSD, (2) determine the relationship between the maternal MTHFD1 gene polymorphisms and the risk of VSD, and 3) investigate the interaction effect of maternal MTHFD1 gene polymorphisms and dietary habits on the risk of VSD.

\section{MATERIALS AND METHODS}

\section{Recruitment of Study Participants}

The recruitment was conducted by the Hunan Provincial Children's Hospital (Changsha, Hunan Province, China) from November 2017 to December 2019. A case-control design was adopted in the present study. Mothers of infants with VSD were assigned to the case group, whereas mothers of infants who did not have a congenital malformation following medical examination were assigned to the control group. 
Participants in the research were recruited from two distinct clinics within the same hospital. VSD children were consecutively recruited from the Department of Cardiothoracic Surgery, and the control children were randomly recruited from the Department of Child Healthcare after health counseling or medical examination during the same study time as the cases. It is worth noting that the recruiting location we chose is well-known in the province because of its outstanding CHD diagnosis and treatment capabilities. As a result, this hospital's CHD patient source covers the whole province, indicating a good representation of cases. We ensured that all children in the case and control groups were <one 1 year old to decrease maternal recall bias for exposures prior to the current pregnancy and in early pregnancy. We only included individuals of Han Chinese descent because a homogeneous ethnic background may eliminate residual confounding effects from genetic and cultural differences. Thus, all eligible participants should fulfill the inclusion criteria as follows: (1) provided informed consent, (2) completed the questionnaire, (3) singleton pregnancy, (4) spontaneous pregnancy, (5) Han Chinese population, and 6) provided the blood sample. Of note, this study only included non-syndromic VSD; those with syndromic VSD, such as those with additional organ malformations or known abnormalities, were excluded.

Our study was approved by the ethics committee of the Xiangya School of Public Health of Central South University, and written informed consent was obtained from all mothers. Besides this, we have registered this study in the Chinese Clinical Trial Registry Center (registration number: ChiCTR1800016635).

\section{Information Collection}

The outcome of interest in this study was VSD, which was defined using the Chinese Surveillance of Birth Defects Classification System. All VSD cases were diagnosed using echocardiography and confirmed by surgery. One of the main exposures of interest was maternal dietary habits in early pregnancy. Based on prior food frequency surveys and local eating customs, a self-administered food frequency questionnaire was developed $(23,24)$. When children were taken to the hospital for tests or surgery, professionally trained investigators conducted a face-to-face interview with their mothers to fill out this questionnaire reflecting the maternal dietary habits throughout the early pregnancy.

The questionnaire has established content validity, test-retest reliability $(r=0.867)$, and internal consistency $(\alpha=0.811)$. It focuses on maternal dietary habits in terms of pickled vegetables, smoked foods, grilled foods, fried foods, fresh meat, fish, and shrimp, fresh eggs, fresh vegetables or fruits, soy foods, and milk products. In this study, the frequency of food consumption was defined as follows: (1) hardly intake was defined as less than or equal to two times per week; (2) sometimes intake was defined as three to five times per week; and (3) often intake was defined as more than or equal to six times per week.

In consideration of potential confounders, the questionnaire also included several characteristics as covariates, and the following information was preselected: age at pregnancy onset $(<25,25-29,30-34$, or $\geq 35)$, family income in the past 1 year
$(<50,000,50,000-100,000,100,000-150,000, \geq 150,000 R M B)$, residence (rural or urban), maternal education level ( $<9,9-12$, $13-16$, or $\geq 17$ years), maternal pre-pregnancy body mass index (BMI) (<18.5, 18.5-23.9, 24-26.9, or $\geq 27$ ), active smoking (do you have a smoking experience in 3 months before pregnancy and/or during the first-trimester pregnancy, yes or no), drink (do you have a drinking alcohol experience in 3 months before pregnancy and/or during the first-trimester pregnancy, yes or no), folic acid supplementation use (do you use any folic acid supplementation in 3 months before pregnancy and/or during the first-trimester pregnancy, yes or no), antibiotic use (do you use any antibiotic (i.e., $\beta$-Lactams, aminoglycosides, macrolides, tetracycline, quinolones, and chloramphenicol) in the 3 months before pregnancy and/or during the first-trimester pregnancy, yes or no), gestational diabetes (yes or no), gestational hypertension (yes or no), history of congenital heart diseases in family (yes or no), and consanguineous marriage (yes or no).

\section{SNP Selection and Genotyping}

When mothers completed the abovementioned questionnaires, they were asked to provide 3 to $5 \mathrm{ml}$ of peripheral venous blood for genotyping. Blood samples were collected in EDTAtreated (ethylenediamine tetraacetic acid) anticoagulant tubes immediately after blood sampling and centrifuged to separate plasma and blood cells. Blood cells were isolated and kept at $-80^{\circ} \mathrm{C}$ until genotyping. The MTHFD1 gene candidate loci were chosen based on earlier published research that indicated MTHFD1 gene polymorphisms (22) and their interactions with maternal dietary habits influenced developmental abnormalities such as CHD $(16,21)$. As a result, rs1950902, rs2236225, and rs2236222 were eventually chosen as candidate loci in our study.

Briefly, SNP markers were selected using the SNPBrowser ${ }^{\mathrm{TM}}$ program (version 3.0) provided by AppliedBiosystems Inc. This program allowed selection of SNP markers from the HapMap database (http://www.hapmap.org/). We excluded these SNPs with minor allele frequencies $<10 \%$ in Caucasians. The polymorphisms of the MTHFD1 gene were tested using a matrix-assisted laser desorption and ionization time-of-flight mass spectrometry MassARRAY system (Agena iPLEX assay, San Diego, CA, USA). The lab technicians who did the genotyping, retyped and double-checked each sample, and recorded the genotype data were unaware of whether the samples were from cases or controls. We imposed a minimum SNP genotyping call rate at the level of $50 \%$, which was applied to ensure data integrity of the individual's genotypes that had been called. Successful rates for SNPlex assays were all $>90 \%$ for the three SNPs.

\section{Statistical Analysis}

Statistical analysis was performed using R software, version 3.5.0 (R Foundation for Statistical Computing). All tests were twotailed, and $P<.05$ was considered to indicate a statistically significant difference except where otherwise specified. To minimize type I error, a false discovery rate $P$ value (FDR_P) was applied to multiple test corrections based on BenjaminiHochberg. The statistically significant results should meet FDR_P $<0.05$ if applicable. 
The distribution of the individual's baseline characteristics in the study population was presented as a number (proportion) for categorical data. Whether or not the differences between cases and controls were substantial was tested with chi-square for categorical variables. Hardy-Weinberg equilibrium (HWE) was tested for the control group (significance level at $P<0.05$ ). We comprehensively analyzed the association of genotype and three genetic models (i.e., dominant, recessive, and additive models) for every SNP with the risk of VSD. The dominant model meant heterozygote and mutant type homozygote vs. wild type homozygote, the recessive model meant mutant type homozygote vs. heterozygote and wild type homozygote, the additive model meant mutant type homozygote vs. heterozygote vs. mutant type homozygote.

Odds ratios (OR) and their 95\% confidence intervals (CIs) were used to show the strength of association. The main effect of maternal dietary habits and MTHFD1 gene polymorphisms was estimated by aOR in multivariate logistic regression models to control for potential confounders. Furthermore, the interaction effect of MTHFD1 gene polymorphisms and maternal dietary habits was also examined by logistic regression models.

\section{RESULTS}

\section{Characteristics of Study Participants}

In this study, we recruited 864 eligible participants, including 360 mothers of infants with VSD in the case group and 504 mothers of children without a congenital malformation in the control group. Among 360 children with VSD, 50 (13.9 \%) had atrial septal defect, 98 (27.2\%) had patent ductus arteriosus, $6(1.7 \%)$ had aorto-pulmonary window, $2(0.6 \%)$ had complete transposition of great arteries, and 24 (6.7\%) had tetralogy of Fallot, but 206 (57.2\%) had no other subtypes of CHD. Considering that some cases were diagnosed with multiple subtypes of CHD, the sum of the various subtypes was not equal to 360 . Comparisons of baseline characteristics across the two groups are summarized in Table 1. Our study shows that there are statistically significant differences between groups for the following characteristics: age at pregnancy onset, residence, maternal education level, active smoking, drink, folic acid use, gestational diabetes, gestational hypertension, history of congenital heart diseases in family, and consanguineous marriage (all $P$ values $<0.05$ ). However, we did not observe statistically significant differences in family income in the past 1 year $(P=0.100)$, antibiotic use $(P=0.092)$, and pre-pregnancy BMI $(P=0.108)$ between the case and control groups. As a result, when evaluating the associations of maternal dietary habits, SNPs of MTHFD1 gene polymorphisms, and their interactions with the risk of VSD in offspring, these statistically significant factors were adjusted as confounders.

\section{Maternal Dietary Habits and the Risk of VSD in Offspring}

Table 2 shows the frequency of maternal dietary intake throughout early pregnancy. Pickled vegetables, smoked foods, grilled foods, fried foods, fresh meat, fish and shrimp, fresh eggs, fresh fruits or vegetables, soy foods, and milk products were observed to be significantly associated with the risk of
VSD in offspring (all $P$ values $<0.05$ ). To control for potential confounders between the two groups, we used multivariate logistic regression to assess the associations of maternal dietary habits with the risk of VSD (Table 2). Maternal excessive intake of pickled vegetables $(\mathrm{aOR}=1.85,95 \% \mathrm{CI}$ : 1.45-2.37), smoked foods $(\mathrm{aOR}=1.93,95 \% \mathrm{CI}: 1.48-2.51)$, barbecued foods $(\mathrm{aOR}=$ 1.74, 95\%CI: $1.28-2.36$ ), and fried foods ( $\mathrm{aOR}=1.68,95 \% \mathrm{CI}$ : $1.30-2.17)$ were shown to be associated with a higher risk of VSD in offspring. In contrast, we observed that maternal excessive intake of fresh meat $(\mathrm{aOR}=0.61,95 \% \mathrm{CI}: 0.47-0.79)$, fish and shrimp ( $\mathrm{aOR}=0.29$, 95\%CI: 0.23-0.38), fresh eggs $(\mathrm{aOR}=0.54$, 95\%CI: $0.42-0.70)$, fresh fruits or vegetables $(\mathrm{aOR}=0.44,95 \% \mathrm{CI}$ : $0.33-0.60$ ), soy foods (aOR $=0.65,95 \% \mathrm{CI}: 0.53-0.80)$, and milk products $(\mathrm{aOR}=0.49,95 \% \mathrm{CI}: 0.40-0.59)$ could significantly contribute to a lower risk of VSD in offspring.

\section{Maternal MTHFD1 Gene Polymorphisms and the Risk of VSD in Offspring}

The genotype frequencies of the MTHFD1 gene at rs1950902, rs2236222, and rs2236225 were all within HWE (all $P$ values $>$ $0.05)$, according to the results of HWE tests in the control group (Table 3).

According to the univariate analyses (Table 4), the genetic polymorphisms of MTHFD1 gene at rs1950902 (the dominant model: FDR_P $=0.008$ ) and rs2236222 (the dominant model: FDR_P $=0.036)$ between the case and control groups were statistically significant. Taking into account potential confounders, we then applied multivariate logistic regression to determine the associations of MTHFD1 gene polymorphisms on the risk of VSD (Table 4). After adjustment for baseline data, the genetic polymorphisms of the maternal MTHFD1 gene at rs1950902 were shown to be significantly associated with a lower risk of VSD in offspring (GA vs. GG: $\mathrm{aOR}=0.67$, 95\%CI: $0.50-0.90$; the dominant model: aOR $=0.66,95 \% \mathrm{CI}$ : $0.50-0.88$; the additive model: $\mathrm{aOR}=0.76,95 \% \mathrm{CI}: 0.62-0.93$ ). Additionally, the genetic polymorphisms of maternal MTHFD1 gene at rs2236222 were significantly associated with a higher risk of VSD in offspring (GG vs. AA: aOR $=2.75$, 95\%CI: $1.57-$ 4.83; the dominant model: $\mathrm{aOR}=1.44,95 \% \mathrm{CI}$ : $1.09-1.90$; the recessive model: $\mathrm{aOR}=2.52,95 \% \mathrm{CI}: 1.45-4.38$; the additive model: $\mathrm{aOR}=1.46,95 \% \mathrm{CI}: 1.17-1.82)$. However, we found no statistically significant associations between maternal MTHFD1 gene polymorphisms at rs2236225 and the risk of VSD.

\section{Interaction Effects of Maternal Dietary Habits and the MTHFD1 Gene on the Risk of VSD in Offspring}

Multivariate logistic regression was used to determine if maternal dietary habits and MTHFD1 gene polymorphisms had statistically significant interaction effects on the development of VSD in children (Table 5). Multivariate logistic regression was applied to determine if maternal dietary habits and MTHFD1 gene polymorphisms had statistically significant interaction effects on the development of VSD in offspring (Table 5). For rs1950902, significant interactions of the variant genotypes $(\mathrm{GA}+\mathrm{AA})$ and excessive intake of fresh meat $(\mathrm{aOR}=0.84$, 
TABLE 1 | Baseline characteristics in case and control groups ${ }^{\text {a }}$.

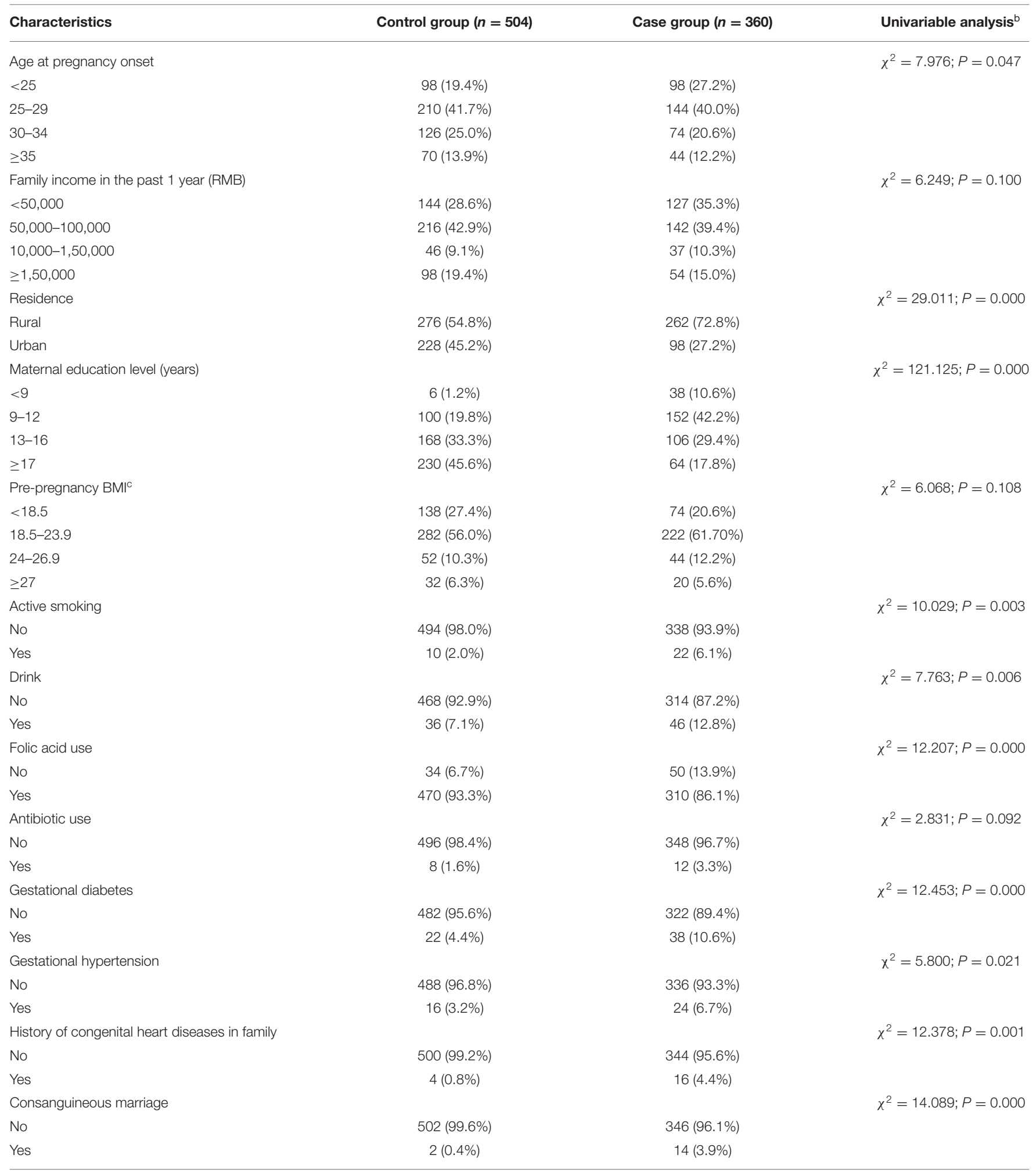

BMI, body mass index.

${ }^{a}$ Data presented as number (percentage) unless otherwise indicated.

${ }^{b} P<0.05$ was considered to indicate a statistically significant difference.

${ }^{c}$ Classification according to Chinese standard for obesity BMI. 
TABLE 2 | Maternal dietary habits and the risk of VSD in offspringa

\begin{tabular}{|c|c|c|c|c|c|}
\hline Maternal dietary habits & Control group $(n=504)$ & Case group $(n=360)$ & Univariate analysis & Unadjusted OR (95\% Cl) & Adjusted OR $(95 \% \mathrm{Cl})^{\mathrm{b}}$ \\
\hline Pickled vegetables & & & $\chi^{2}=18.382 ; P=0.000$ & $1.60(1.29-1.98)$ & $1.85(1.45-2.37)$ \\
\hline Hardly & $330(65.5 \%)$ & $188(52.2 \%)$ & & 1 & 1 \\
\hline Sometimes & $148(29.4 \%)$ & $134(37.2 \%)$ & & $1.59(1.18-2.13)$ & $1.87(1.34-2.61)$ \\
\hline Often & $26(5.2 \%)$ & $38(10.6 \%)$ & & $2.57(1.51-4.36)$ & $3.40(1.87-6.17)$ \\
\hline Smoked foods & & & $\chi^{2}=20.562 ; P=0.000$ & $1.69(1.34-2.13)$ & $1.93(1.48-2.51)$ \\
\hline Hardly & $276(54.8 \%)$ & $149(41.4 \%)$ & & 1 & 1 \\
\hline Sometimes & $212(42.1 \%)$ & $182(50.6 \%)$ & & $1.59(1.20-2.11)$ & $1.91(1.38-2.63)$ \\
\hline Often & $16(3.2 \%)$ & $29(8.1 \%)$ & & $3.36(1.77-6.38)$ & $3.82(1.87-7.81)$ \\
\hline Barbecued foods & & & $\chi^{2}=18.455 ; P=0.000$ & $1.77(1.35-2.31)$ & $1.74(1.28-2.36)$ \\
\hline Hardly & 376 (74.6\%) & 225 (62.5\%) & & 1 & 1 \\
\hline Sometimes & $124(24.6 \%)$ & $123(34.2 \%)$ & & $1.66(1.23-2.24)$ & $1.70(1.21-2.38)$ \\
\hline Often & $4(0.8 \%)$ & $12(3.3 \%)$ & & $5.01(1.6-15.73)$ & $3.66(1.06-12.57)$ \\
\hline Fried foods & & & $\chi^{2}=15.235 ; P=0.000$ & $1.53(1.23-1.91)$ & $1.68(1.30-2.17)$ \\
\hline Hardly & $306(60.7 \%)$ & $180(50 \%)$ & & 1 & 1 \\
\hline Sometimes & $178(35.3 \%)$ & $147(40.8 \%)$ & & $1.40(1.06-1.87)$ & $1.82(1.31-2.53)$ \\
\hline Often & $20(4 \%)$ & $33(9.2 \%)$ & & $2.81(1.56-5.04)$ & $2.40(1.25-4.58)$ \\
\hline Fresh meat & & & $\chi^{2}=28.493 ; P=0.000$ & $0.55(0.43-0.69)$ & $0.61(0.47-0.79)$ \\
\hline Hardly & $22(4.4 \%)$ & $53(14.7 \%)$ & & 1 & 1 \\
\hline Sometimes & $20(4 \%)$ & $14(3.9 \%)$ & & $0.29(0.13-0.68)$ & $0.27(0.10-0.68)$ \\
\hline Often & $462(91.7 \%)$ & $293(81.4 \%)$ & & $0.26(0.16-0.44)$ & $0.32(0.18-0.56)$ \\
\hline Fish and shrimp & & & $\chi^{2}=154.281 ; P=0.000$ & $0.25(0.20-0.32)$ & $0.29(0.23-0.38)$ \\
\hline Hardly & $22(4.4 \%)$ & $80(22.2 \%)$ & & 1 & 1 \\
\hline Sometimes & $146(29 \%)$ & $186(51.7 \%)$ & & $0.35(0.21-0.59)$ & $0.38(0.22-0.66)$ \\
\hline Often & $336(66.7 \%)$ & $94(26.1 \%)$ & & $0.08(0.05-0.13)$ & $0.10(0.06-0.18)$ \\
\hline Fresh eggs & & & $\chi^{2}=58.659 ; P=0.000$ & $0.44(0.35-0.56)$ & $0.54(0.42-0.70)$ \\
\hline Hardly & $24(4.8 \%)$ & $44(12.2 \%)$ & & 1 & 1 \\
\hline Sometimes & 56 (11.1\%) & $96(26.7 \%)$ & & $0.94(0.52-1.70)$ & $1.07(0.55-2.07)$ \\
\hline Often & $424(84.1 \%)$ & $220(61.1 \%)$ & & $0.28(0.17-0.48)$ & $0.41(0.23-0.73)$ \\
\hline Fresh fruits or vegetables & & & $\chi^{2}=70.061 ; P=0.000$ & $0.38(0.29-0.50)$ & $0.44(0.33-0.60)$ \\
\hline Hardly & $10(2 \%)$ & $66(18.3 \%)$ & & 1 & 1 \\
\hline Sometimes & $26(5.2 \%)$ & 14 (3.9\%) & & $0.08(0.03-0.21)$ & $0.07(0.03-0.19)$ \\
\hline Often & 468 (92.9\%) & $280(77.8 \%)$ & & $0.09(0.05-0.18)$ & $0.12(0.06-0.24)$ \\
\hline Soy foods & & & $\chi^{2}=53.235 ; P=0.000$ & $0.52(0.43-0.62)$ & $0.65(0.53-0.80)$ \\
\hline Hardly & 70 (13.9\%) & $107(29.7 \%)$ & & 1 & 1 \\
\hline Sometimes & $146(29 \%)$ & 131 (36.4\%) & & $0.59(0.40-0.86)$ & $0.72(0.47-1.10)$ \\
\hline Often & $288(57.1 \%)$ & $122(33.9 \%)$ & & $0.28(0.19-0.40)$ & $0.43(0.28-0.65)$ \\
\hline Milk products & & & $\chi^{2}=125.216 ; P=0.000$ & $0.43(0.36-0.50)$ & $0.49(0.40-0.59)$ \\
\hline Hardly & $82(16.3 \%)$ & 187 (51.9\%) & & 1 & 1 \\
\hline Sometimes & $120(23.8 \%)$ & 55 (15.3\%) & & $0.20(0.13-0.30)$ & $0.23(0.15-0.37)$ \\
\hline Often & 302 (59.9\%) & 118 (32.8\%) & & $0.17(0.12-0.24)$ & $0.23(0.15-0.33)$ \\
\hline
\end{tabular}

VSD, ventricular septal defect; OR, odds ratio; $\mathrm{Cl}$, confidence interval.

${ }^{a}$ Data presented as number (percentage) unless otherwise indicated.

${ }^{b}$ Adjusted for age at pregnancy onset, residence, education level, active smoking, drink, folic acid use, gestational diabetes, gestational hypertension, history of congenital heart diseases in family and consanguineous marriage.

95\%CI: 0.75-0.93), fish and shrimp (aOR $=0.76$, 95\%CI: $0.68-$ $0.86)$, fresh eggs $(\mathrm{aOR}=0.85,95 \% \mathrm{CI}: 0.76-0.95)$, fruits or vegetables $(\mathrm{aOR}=0.82,95 \% \mathrm{CI}$ : $0.74-0.91)$, soy foods $(\mathrm{aOR}=$ $0.82,95 \% \mathrm{CI}: 0.72-0.93$ ), and milk products $(\mathrm{aOR}=0.77,95 \% \mathrm{CI}$ : $0.68-0.87$ ) were observed.

With regard to rs2236225, the interactions of the variant genotypes (GA+AA) with excessive intake of pickled vegetables
$(\mathrm{aOR}=1.34,95 \% \mathrm{CI}: 1.11-1.61)$ and fried foods $(\mathrm{aOR}=1.32$, 95\%CI: 1.09-1.59) were shown to be statistically significant.

For rs2236222, we found significant interactions between the variant genotypes $(\mathrm{GA}+\mathrm{GG})$ and excessive intake of pickled vegetables $(\mathrm{aOR}=1.44,95 \% \mathrm{CI}: 1.19-1.74)$, smoked foods $(\mathrm{aOR}$ $=1.37,95 \% \mathrm{CI}: 1.14-1.63)$, barbecued foods $(\mathrm{aOR}=1.39,95 \% \mathrm{CI}$ : $1.12-1.72$ ), and fried foods ( $\mathrm{aOR}=1.27,95 \% \mathrm{CI}: 1.06-1.53)$. 
TABLE 3 | Genotypes Frequencies of genetic loci of MTHFD1 gene and Hardy-Weinberg Disequilibrium tests of the control groupa.

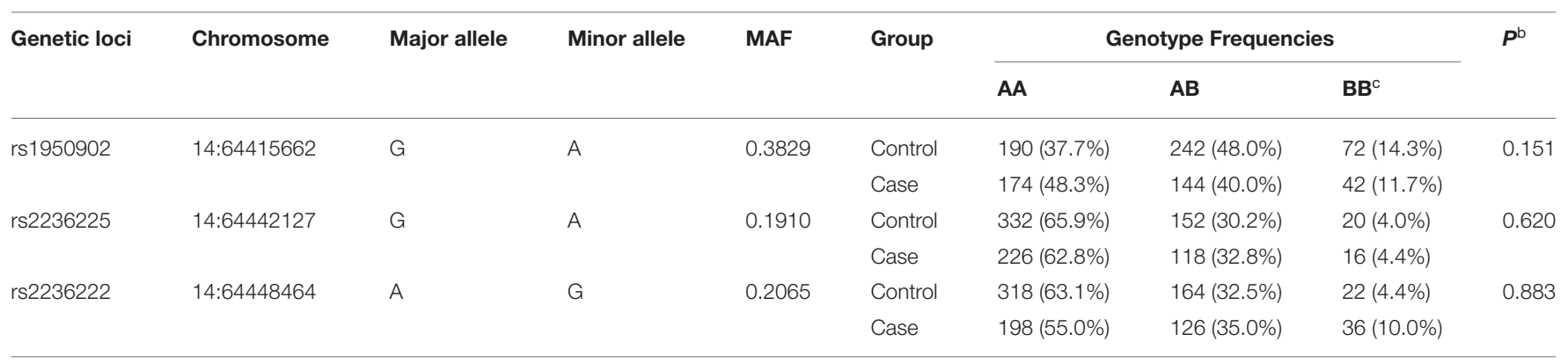

MTHFD1, Methylenetetrahydrofolate dehydrogenase 1; MAF, minimum allele frequency.

${ }^{a}$ Data presented as number (percentage) unless otherwise indicated.

${ }^{b} P<0.05$ was considered to indicate a statistically significant difference.

${ }^{c} A A$, Homozygous with minor allele.

$A B$, Heterozygous.

$B B$, Homezygous with major allele.

\section{DISCUSSION}

Previous studies concentrate on the involvement of particular nutrients in the etiology of $\mathrm{CHD}(18,19)$. In fact, when considering the interactions in distinct nutrients, maternal dietary habits were more appropriate for assessing associations between dietary factors and CHD than a specific nutrient or hazardous substance separately. Based on a case-control study, we, therefore, focused on the associations between maternal dietary habits and the risk of VSD in offspring. Our findings suggest that mothers who adhered to excessive intakes of pickled vegetables $(\mathrm{aOR}=1.85)$, smoked foods $(\mathrm{aOR}=1.93)$, barbecued foods $(\mathrm{aOR}=1.74)$, and fried foods $(\mathrm{aOR}=$ 1.68) during their early pregnancy were more likely to have a VSD-affected infant.

Pickled vegetables contain relatively large amounts of nitrite and $\mathrm{N}$-nitroso compounds. Approximately $85 \%$ of dietary nitrate was derived from vegetables with the remaining $15 \%$ derived primarily from drinking water (25). It was demonstrated that maternally consumed nitrate/nitrite was capable of permeating the placenta and affecting the developing fetus. Also, previous epidemiologic studies show the positive associations between maternal exposure to nitrate, nitrite, and $\mathrm{N}$-nitroso compounds from diet or drinking water and an increased risk of congenital abnormalities, such as neural tube defects, oral cleft malformations, limb deficiencies, and $\operatorname{CHD}(25,26)$, which provided evidence for our findings. Additionally, smoked, barbecued, and fried foods were a common dietary source of polycyclic aromatic hydrocarbons (PAHs). Because of their capacity to easily cross cellular membranes, including the embryonic and fetal blood-brain barrier, PAHs were considered teratogens, resulting in increased oxidative stress and DNA damage (27). A variety of birth outcomes and anomalies are reported as a result of PAH exposure, and evidence suggests that maternal exposure to PAHs during early pregnancy is linked to CHD (28), which may partially explain our observation of a positive association between maternal excessive intakes of smoked, barbecued, and fried foods and an increased VSD risk in offspring.
In this study, we also found that maternal excessive consumption of fish and shrimp $(\mathrm{aOR}=0.29)$, fresh fruits or vegetables $(\mathrm{aOR}=0.44)$, milk products $(\mathrm{aOR}=0.49)$, fresh meat $(\mathrm{aOR}=0.61)$, and soy foods $(\mathrm{aOR}=0.65)$ during early pregnancy was significantly associated with a reduced risk of VSD in offspring. Fish and shrimp, fresh fruits or vegetables, soy foods, and milk products are all common food types that are rich in proteins, lipids, vitamins, and minerals and provide many essential amino acids and fatty acids for growth and maturation. Of note, among all common food types, fish and shrimp have the strongest preventive benefits against the development of VSD. According to one study conducted by Obermann-Borst (21), a high intake of fish and seafood, which belong to the one-carbon-rich dietary pattern, supplied total protein; vitamins $\mathrm{B} 1, \mathrm{~B} 2, \mathrm{~B} 3, \mathrm{~B} 6$, and B12; zinc; EPA; and DHA, which was related to a lower risk of CHD in offspring. Zinc serves as a cofactor for -glutamylhydrolase, a folate absorption enzyme, and methionine synthase, a homocysteine-to-methionine conversion enzyme (29). A moderate amount of intake of zinc is proved to be linked with a reduced risk of CHD in the offspring (30). Also, high levels of omega-3 fatty acid (EPA and DHA) intake from fish have a homocysteine-lowering effect, which may explain some of the beneficial effects of fish intake on pregnancy outcome (31).

To date, the role of MTHFR gene polymorphisms in birth defects has received a great attention, particularly the C667T variant. However, other essential genes in the folate metabolism pathway, such as the MTHFD1 gene, also play a crucial role in maintaining the folate-homocysteine balance and should not be overlooked. Experimental evidence suggests that the MTHFD1 gene can influence embryonic development by altering folate metabolism. Disruption of the Mthfd1 gene in homozygous mice resulted in early embryonic lethality, whereas mice heterozygous for the disruption appeared healthy but had impaired folatemediated one-carbon metabolism (32). It is assumed that maternal MTHFD1 gene polymorphisms are associated with the risk of VSD in offspring. To provide more epidemiological data, we assessed their associations in Chinese populations.

This study concentrated on three SNPs of the MTHFD1 gene, including two functional non-synonymous polymorphisms 
TABLE 4 | Associations between maternal polymorphisms of MTHFD1 gene and the risk of VSD.

\begin{tabular}{|c|c|c|c|c|}
\hline SNPs & \multicolumn{2}{|c|}{ Univariate logistic regression } & \multicolumn{2}{|c|}{ Multivariate logistic regression } \\
\hline $\mathrm{G} / \mathrm{G}$ & 1.00 & & 1.00 & \\
\hline $\mathrm{G} / \mathrm{A}$ & $0.65(0.49-0.87)$ & 0.012 & $0.67(0.50-0.90)$ & 0.021 \\
\hline A/A & $0.64(0.41-0.98)$ & 0.077 & $0.65(0.42-1.00)$ & 0.098 \\
\hline Recessive model $^{d}$ & $0.79(0.53-1.19)$ & 0.395 & $0.80(0.53-1.20)$ & 0.417 \\
\hline Additive model ${ }^{e}$ & $0.75(0.61-0.92)$ & 0.013 & $0.76(0.62-0.93)$ & 0.021 \\
\hline \multicolumn{5}{|l|}{ rs2236225 } \\
\hline $\mathrm{G} / \mathrm{G}$ & 1.00 & & 1.00 & \\
\hline $\mathrm{G} / \mathrm{A}$ & $1.14(0.85-1.53)$ & 0.440 & $1.15(0.86-1.55)$ & 0.469 \\
\hline Additive model & $1.12(0.88-1.42)$ & 0.440 & $1.09(0.86-1.39)$ & 0.554 \\
\hline \multicolumn{5}{|l|}{ rs2236222 } \\
\hline A/A & 1.00 & & 1.00 & \\
\hline$A / G$ & $1.23(0.92-1.65)$ & 0.265 & $1.27(0.94-1.70)$ & 0.195 \\
\hline $\mathrm{G} / \mathrm{G}$ & $2.63(1.50-4.60)$ & 0.005 & $2.75(1.57-4.83)$ & 0.000 \\
\hline Dominant model & $1.40(1.06-1.84)$ & 0.036 & $1.44(1.09-1.90)$ & 0.021 \\
\hline Recessive model & $2.43(1.41-4.21)$ & 0.005 & $2.52(1.45-4.38)$ & 0.005 \\
\hline Additive model & $1.43(1.15-1.78)$ & 0.005 & $1.46(1.17-1.82)$ & 0.005 \\
\hline
\end{tabular}

VSD, ventricular septal defect; MTHFD1, Methylenetetrahydrofolate dehydrogenase 1; OR, odds ratio; Cl, confidence interval; FDR_P, false discovery rate P value.

${ }^{a} F D R \_P<0.05$ was considered to indicate a statistically significant difference.

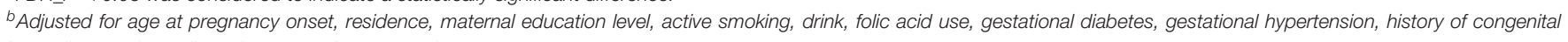
heart diseases in family and consanguineous marriage.

${ }^{c}$ The dominant model means heterozygote and mutant type homozygote vs. wild type homozygote.

${ }^{d}$ The recessive model means mutant type homozygote vs. heterozygote and wild type homozygote.

e The additive model means mutant type homozygote vs. heterozygote vs. mutant type homozygote.

with known biochemical phenotypes, rs1950902 and rs2236225, and one synonymous polymorphism, rs2236222. The rs2236225 polymorphism is the well-studied genetic variant of the MTHFD1 gene, and this SNP has been a hot spot for research into genetic risk factors for birth defects. At nucleotide 1958, MTHFD1 rs2236225 (G1958A; R653Q) undergoes a G-toA change, resulting in an arginine-to-glutamate substitution at amino acid 653. The rs1950902 polymorphism (C401T; $\mathrm{R} 134 \mathrm{~K}$ ), which includes a C-to-T transition at nucleotide 401 and results in an arginine to lysine change at amino acid 134 , is another well-studied genetic variation of the MTHFD1 gene. However, among epidemiological studies, the association between MTHFD1 gene polymorphisms and the risk of VSD has yielded conflicting results. Our findings, as well as Karen (14) and Huang's results (33), suggest that maternal genetic polymorphisms of MTHFD1 gene at rs2236225 could not affect the susceptibility to $\mathrm{CHD}$ in offspring. With respect to the subtypes of CHD, positive associations of MTHFD1 rs2236225 with atrial septal defects (34), tetralogy of Fallot (35), and aortic stenosis (14) were observed. When it came to the polymorphism rs1950902, there were no epidemiologic studies concerning the association between rs1950902 and
VSD but one study involving tetralogy of Fallot. However, in one study by $\mathrm{Xu}$, no evidence for the association between MTHFD1 1950902 and tetralogy of Fallot was found (33). These observations need to be replicated, and further investigations are warranted.

In addition, we found a significant interaction between the maternal MTHFD1 gene and dietary habits on the risk of VSD. This finding seems to be biologically plausible. Previous research has discovered strong connections between dietary habits and folate levels (36). Mothers who consumed comparatively more fish and seafood had higher serum and red blood cell folate levels as compared with those who consumed less fish and seafood (21). Furthermore, Christensen et al. (22) found that low dietary folate interacted with MTHFD1 synthetase deficiency in mice, a model for the R653Q mutation, to greatly increase the risk of birth defects. Nonetheless, the specific mechanism remained unclear, necessitating more research.

The limitations of the study needed to be addressed. First, given that this was hospital-based case-control research, the case and control groups from various clinics in one hospital did not appear to agree on the majority of characteristics. 
TABLE 5 | Interactions of maternal polymorphisms of MTHFD1 gene and maternal dietary habits by multivariate logistic regression.

\begin{tabular}{|c|c|c|c|c|c|c|}
\hline \multirow[t]{2}{*}{ Dietary habits ${ }^{a}$} & \multicolumn{2}{|c|}{ Interaction with rs $1950902^{\mathrm{b}}$} & \multicolumn{2}{|c|}{ Interaction with rs2236225 b } & \multicolumn{2}{|c|}{ Interaction with rs $2236222^{b}$} \\
\hline & aOR $(95 \% \mathrm{CI})^{\mathrm{d}}$ & FDR_Pc & aOR $(95 \% \mathrm{Cl})^{\mathrm{d}}$ & FDR_P $P^{\mathrm{c}}$ & aOR $(95 \% \mathrm{Cl})^{\mathrm{d}}$ & FDR_Pc \\
\hline Pickled vegetables & $0.96(0.81-1.15)$ & 0.686 & $1.34(1.11-1.61)$ & 0.020 & $1.44(1.19-1.74)$ & 0.000 \\
\hline Smoked foods & 0.95 (0.80-1.14) & 0.653 & $1.18(0.98-1.42)$ & 0.208 & $1.37(1.14-1.63)$ & 0.005 \\
\hline Barbecued foods & 0.89 (0.72-1.09) & 0.355 & $1.28(1.04-1.58)$ & 0.073 & $1.39(1.12-1.72)$ & 0.010 \\
\hline Fried foods & $0.91(0.76-1.08)$ & 0.355 & $1.32(1.09-1.59)$ & 0.025 & 1.27 (1.06-1.53) & 0.025 \\
\hline Fresh meat & $0.84(0.75-0.93)$ & 0.002 & $1.02(0.91-1.14)$ & 0.923 & $1.08(0.97-1.21)$ & 0.238 \\
\hline Fish and shrimp & $0.76(0.68-0.86)$ & 0.000 & $0.93(0.82-1.06)$ & 0.550 & $1.00(0.88-1.14)$ & 0.967 \\
\hline Fresh eggs & 0.85 (0.76-0.95) & 0.007 & $0.99(0.88-1.11)$ & 0.923 & $1.10(0.98-1.23)$ & 0.198 \\
\hline Fruits or vegetables & $0.82(0.74-0.91)$ & 0.000 & $1.01(0.90-1.12)$ & 0.923 & $1.07(0.96-1.19)$ & 0.305 \\
\hline Soy foods & $0.82(0.72-0.93)$ & 0.002 & $1.01(0.88-1.15)$ & 0.923 & 1.08 (0.95-1.23) & 0.305 \\
\hline Milk products & $0.77(0.68-0.87)$ & 0.000 & $0.94(0.83-1.08)$ & 0.635 & $0.99(0.87-1.13)$ & 0.967 \\
\hline
\end{tabular}

MTHFD1, Methylenetetrahydrofolate dehydrogenase 1; aOR, adjusted odds ratio; Cl, confidence interval; FDR_P, false discovery rate $P$ value.

${ }^{a}$ Maternal dietary habits were classified as hardly and sometimes/often.

${ }^{b}$ Single nucleotide polymorphisms were classified as wild type and variant genotype.

${ }^{c} F D R \_P<0.05$ was considered to indicate a statistically significant difference.

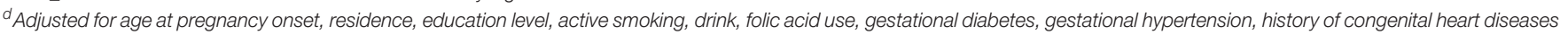
in family and consanguineous marriage.

However, we adjusted the baseline characteristics when exploring the associations of maternal dietary habits, MTHFD1 gene polymorphisms, and their interaction with the risk of VSD in offspring. Second, in consideration of population stratification bias in epidemiologic studies, we recruited the participants restricted to the Han Chinese ethnicity. Due to the obvious ethnic and regional differences in gene polymorphisms, it was necessary to conduct this study in larger and different ethnic populations. Third, because maternal dietary habits during the period of gestation were retrospectively reported by the mother, recall bias was unavoidable. However, to minimize the recall bias, this study only recruited infants who were less one year old in both the case and control groups. One study revealed dietary habits of pregnant women were able to be well-recalled after birth (37). Fourth, this study is mainly concerned with the influence of maternal MTHFD1 gene polymorphisms on the risk of VSD, whereas the role of the polymorphisms of children was ignored. Further research into the associations between MTHFD1 gene polymorphisms in children and VSD risk is required, which might provide additional crucial insights into the etiology of VSD. Last but not least, due to the limitation of the lack of replication studies, it is still to be further studied whether our findings can be extended to different populations.

\section{CONCLUSION}

In this case-control study based on Han Chinese populations, maternal MTHFD1 rs1950902 and rs2236222 were found to be associated with the risk of VSD, but there was no statistically significant association between maternal MTHFD1 rs2236225 and the risk of VSD in offspring. Furthermore, maternal dietary habits as well as their interactions with maternal MTHFD1 gene polymorphisms, have a significant impact in the development of VSD. Due to several limitations in this research, future studies with a larger sample size, a prospective methodology, and different ethnicities are required to corroborate our findings.

\section{DATA AVAILABILITY STATEMENT}

The raw data supporting the conclusions of this article will be made available by the authors, without undue reservation.

\section{ETHICS STATEMENT}

This study was performed in line with the principles of the Declaration of Helsinki. Approval was granted by the Ethics Committee of Xiangya School of Public Health Central South University (No. XYGW-2018-36). The patients/participants provided their written informed consent to participate in this study.

\section{AUTHOR CONTRIBUTIONS}

JD, JL, and YLi performed the experiments. SZ and TW analyzed the data and statistical analyses. JS, YLiu, and MS contributed reagents, material, and analysis tools. XS, PZ, and JQ wrote the main manuscript text. LC, JW, and MS collected reference and managed data. All authors contributed to the article and approved the submitted version.

\section{FUNDING}

This study was supported by the Project Funded by National Natural Science Foundation Program of China (82073653 and 81803313), Hunan Provincial Key Research and Development Program (2018SK2063 and 2018SK2062), Hunan Provincial Science and Technology Talent Support Project (2020TJ-N07), 
Natural Science Foundation of Hunan Province (2018JJ2551), Open Project from NHC Key Laboratory of Birth Defect for Research and Prevention (KF2020006), and the 68th General Program Supported by China Postdoctoral Science Foundation (2020M682644).

\section{REFERENCES}

1. Zhao L, Chen L, Yang T, Wang T, Zhang S, Ye Z, et al. Birth prevalence of congenital heart disease in China, 1980-2019: a systematic review and meta-analysis of 617 studies. Eur J Epidemiol. (2020) 35:63142. doi: 10.1007/s10654-020-00653-0

2. Spicer DE, Hsu HH, Co-Vu J, Anderson RH, Fricker FJ. Ventricular septal defect. Orphanet J Rare Dis. (2014) 9:144. doi: 10.1186/s13023-014-0144-2

3. Rosenquist TH. Folate, homocysteine and the cardiac neural crest. Dev Dyn. (2013) 242:201-18. doi: 10.1002/dvdy.23922

4. Taparia S, Gelineau-van Waes J, Rosenquist TH, Finnell RH. Importance of folate-homocysteine homeostasis during early embryonic development. Clin Chem Lab Med. (2007) 45:1717-27. doi: 10.1515/CCLM.2007.345

5. Botto LD, Lin AE, Riehle-Colarusso T, Malik S, Correa A. Seeking causes: Classifying and evaluating congenital heart defects in etiologic studies. Birth Defects Res A Clin Mol Teratol. (2007) 79:714-27. doi: 10.1002/bdra.20403

6. Morikawa Y, Cserjesi P. Cardiac neural crest expression of Hand2 regulates outflow and second heart field development. Circ Res. (2008) 103:14229. doi: 10.1161/CIRCRESAHA.108.180083

7. Sun M, Wang T, Huang P, Diao J, Zhang S, Li J, et al. Association analysis of maternal MTHFR gene polymorphisms and the occurrence of congenital heart disease in offspring. BMC Cardiovasc Disord. (2021) 21:298. doi: 10.1186/s12872-021-02117-Z

8. Li Y, Diao J, Li J, Luo L, Zhao L, Zhang S, et al. Association of maternal dietary intakes and CBS gene polymorphisms with congenital heart disease in offspring. Int. J. Cardiol. (2021) 322:121-8. doi: 10.1016/j.ijcard.2020.08.018

9. Yu D, Yang L, Shen S, Fan C, Zhang W, Mo X. Association between methionine synthase reductase A66G polymorphism and the risk of congenital heart defects: evidence from eight case-control studies. Pediatr Cardiol. (2014) 35:1091-8. doi: 10.1007/s00246-014-0948-9

10. Hol FA, Put NMVD, Geurds MP, Heil SG, Trijbels FJ, Hamel $\mathrm{BC}$, et al. Molecular genetic analysis of the gene encoding the trifunctional enzyme MTHFD (methylenetetrahydrofolate-dehydrogenase, methenyltetrahydrofolate-cyclohydrolase, formyltetrahydrofolate synthetase) in patients with neural tube defects. Clin Genet. (1998) 53:119-25. doi: 10.1111/j.1399-0004.1998.tb02658.x

11. Christensen KE, MacKenzie RE. Mitochondrial one-carbon metabolism is adapted to the specific needs of yeast, plants and mammals. Bioessays. (2006) 28:595-605. doi: 10.1002/bies.20420

12. Blount BC, Mack MM, Wehr CM, MacGregor JT, Hiatt RA, Wang $\mathrm{G}$, et al. Folate deficiency causes uracil misincorporation into human DNA and chromosome breakage: implications for cancer and neuronal damage. Proc Natl Acad Sci USA. (1997) 94:3290-5. doi: 10.1073/pnas.94. 7.3290

13. Castro R, Rivera I, Ravasco P, Camilo ME, Jakobs C, Blom HJ, et al. 5,10methylenetetrahydrofolate reductase (MTHFR) $677 \mathrm{C}->\mathrm{T}$ and $1298 \mathrm{~A}->\mathrm{C}$ mutations are associated with DNA hypomethylation. J Med Genet. (2004) 41:454-8. doi: 10.1136/jmg.2003.017244

14. Christensen KE, Rohlicek CV, Andelfinger GU, Michaud J, Rozen R. The MTHFD1 p.Arg653Gln Variant Alters Enzyme Function and Increases Risk for Congenital Heart Defects. Hum Mutat. (2009) 30:212-20. doi: 10.1002/humu.20830

15. Christensen KE, Patel H, Kuzmanov U, Mejia NR, MacKenzie RE. Disruption of the mthfd1 gene reveals a monofunctional 10-formyltetrahydrofolate synthetase in mammalian mitochondria. J Biol Chem. (2005) 280:7597602. doi: 10.1074/jbc.M409380200

16. Shaw GM, Lu W, Zhu H, Yang W, Briggs FBS, Carmichael SL, et al. 118 SNPs of folate-related genes and risks of spina bifida and conotruncal heart defects. BMC Med Genet. (2009) 10:49. doi: 10.1186/1471-2350-10-49

\section{ACKNOWLEDGMENTS}

The authors would like to thank the editors and reviewers for their suggestions and all colleagues working in Maternal and Child Health Promotion and Birth Defect Prevention Group.

17. Brody LC, Conley M, Cox C, Kirke PN, McKeever MP, Mills $\mathrm{JL}$, et al. A polymorphism, R653Q, in the trifunctional enzyme methylenetetrahydrofolate dehydrogenase/methenyltetrahydrofolate cyclohydrolase/formyltetrahydrofolate synthetase is a maternal genetic risk factor for neural tube defects: report of the birth defects research group. Am J Hum Genet. (2002) 71:1207-15. doi: 10.1086/344213

18. Smedts HP, de Vries JH, Rakhshandehroo M, Wildhagen MF, VerkleijHagoort AC, Steegers EA, et al. High maternal vitamin E intake by diet or supplements is associated with congenital heart defects in the offspring. BJOG. (2009) 116:416-23. doi: 10.1111/j.1471-0528.2008.01957.x

19. Verkleij-Hagoort AC, de Vries JH, Ursem NT, de Jonge R, Hop WC, Steegers-Theunissen RP. Dietary intake of B-vitamins in mothers born a child with a congenital heart defect. Eur J Nutr. (2006) 45:47886. doi: 10.1007/s00394-006-0622-y

20. Shaw GM, Carmichael SL, Yang W, Lammer EJ. Periconceptional nutrient intakes and risks of conotruncal heart defects. Birth Defects Res A Clin Mol Teratol. (2010) 88:144-51. doi: 10.1002/bdra.20648

21. Obermann-Borst SA, Vujkovic M, de Vries JH, Wildhagen MF, Looman CW, de Jonge R, et al. A maternal dietary pattern characterised by fish and seafood in association with the risk of congenital heart defects in the offspring. BJOG. (2011) 118:1205-15. doi: 10.1111/j.1471-0528.2011.02984.x

22. Christensen KE, Bahous RH, Hou W, Deng L, Malysheva OV, Arning E, et al. Low Dietary folate interacts with MTHFD1 synthetase deficiency in mice, a model for the R653Q variant, to increase incidence of developmental delays and defects. J Nutr. (2018) 148:501-9. doi: 10.1093/jn/nxy013

23. Yang J, Kang Y, Cheng Y, Zeng L, Yan H, Dang S. Maternal dietary patterns during pregnancy and congenital heart defects: a case-control study. Int J Environ Res Public Health. (2019) 16:2957. doi: 10.3390./ijerph161 62957

24. Cheng Y, Yan H, Dibley MJ, Shen Y, Li Q, Zeng L. Validity and reproducibility of a semi-quantitative food frequency questionnaire for use among pregnant women in rural China. Asia Pac J Clin Nutr. (2008) 17:166-77.

25. Blaisdell J, Turyk ME, Almberg KS, Jones RM, Stayner LT. Prenatal exposure to nitrate in drinking water and the risk of congenital anomalies. Environ Res. (2019) 176:108553. doi: 10.1016/j.envres.2019.108553

26. Brender JD, Olive JM, Felkner M, Suarez L, Marckwardt W, Hendricks KA. Dietary nitrites and nitrates, nitrosatable drugs, and neural tube defects. Epidemiology. (2004) 15:330-6. doi: 10.1097/01.ede.0000121381.79831.7b

27. Huang HB, Chen GW, Wang CJ, Lin YY, Liou SH, Lai CH, et al. Exposure to heavy metals and polycyclic aromatic hydrocarbons and DNA damage in taiwanese traffic conductors. Cancer Epidemiol Biomarkers Prev. (2013) 22:102-8. doi: 10.1158/1055-9965.EPI-12-0706

28. Incardona JP, Collier TK, Scholz NL. Defects in cardiac function precede morphological abnormalities in fish embryos exposed to polycyclic aromatic hydrocarbons. Toxicol Appl Pharmacol. (2004) 196:191-205. doi: 10.1016/jtaap11(2003)026

29. Wallwork JC, Duerre JA. Effect of zinc deficiency on methionine metabolism, methylation reactions and protein synthesis in isolated perfused rat liver. $J$ Nutr. (1985) 115:252-62. doi: 10.1093/jn/115.2.252

30. Smedts HP, Rakhshandehroo M, Verkleij-Hagoort AC, de Vries JH, Ottenkamp J, Steegers EA, et al. Maternal intake of fat, riboflavin and nicotinamide and the risk of having offspring with congenital heart defects. Eur J Nutr. (2008) 47:357-65. doi: 10.1007/s00394-008-0735-6

31. Rajaram S, Haddad EH, Mejia A, Sabaté J. Walnuts and fatty fish influence different serum lipid fractions in normal to mildly hyperlipidemic individuals: a randomized controlled study. Am J Clin Nutr. (2009) 89:1657s-63s. doi: 10.3945/ajcn.2009.26736S

32. Sutherland HG, Hermile H, Sanche R, Menon S, Lea RA, Haupt LM, et al. Association study of MTHFD1 coding polymorphisms R134K 
and R653Q with migraine susceptibility. Headache. (2014) 54:150614. doi: $10.1111 /$ head.12428

33. Xu J, Xu X, Xue L, Liu $X, G u H$, Cao $H$, et al. MTHFR c.1793G $>$ A polymorphism is associated with congenital cardiac disease in a Chinese population. Cardiol. Young. (2010) 20:31826. doi: $10.1017 / \mathrm{S} 1047951110000247$

34. Cheng J, Zhu WL, Dao JJ, Li SQ, Li Y. Relationship between polymorphism of methylenetetrahydrofolate dehydrogenase and congenital heart defect. Biomed Environ Sci. (2005) 18:58-64.

35. Huang J, Mei J, Jiang L, Jiang Z, Liu H, Ding F. MTHFR rs1801133 C>T polymorphism is associated with an increased risk of tetralogy of Fallot. Biomedical reports. (2014) 2:172-6. doi: 10.3892/br.2014.222

36. Fischer M, Stronati M, Lanari M. Mediterranean diet, folic acid, and neural tube defects. Ital J Pediatr. (2017) 43:74. doi: 10.1186/s13052-017-0391-7

37. Bunin GR, Gyllstrom ME, Brown JE, Kahn EB, Kushi LH. Recall of diet during a past pregnancy. Am J Epidemiol. (2001) 154:113642. doi: 10.1093/aje/154.12.1136
Conflict of Interest: The authors declare that the research was conducted in the absence of any commercial or financial relationships that could be construed as a potential conflict of interest.

Publisher's Note: All claims expressed in this article are solely those of the authors and do not necessarily represent those of their affiliated organizations, or those of the publisher, the editors and the reviewers. Any product that may be evaluated in this article, or claim that may be made by its manufacturer, is not guaranteed or endorsed by the publisher.

Copyright (c) 2022 Song, Liu, Wang, Zhang, Sun, Shu, Wei, Diao, Li, Li, Chen, Zhu and Qin. This is an open-access article distributed under the terms of the Creative Commons Attribution License (CC BY). The use, distribution or reproduction in other forums is permitted, provided the original author(s) and the copyright owner(s) are credited and that the original publication in this journal is cited, in accordance with accepted academic practice. No use, distribution or reproduction is permitted which does not comply with these terms. 